\author{
MITSUBISHI ELECTRIC RESEARCH LABORATORIES \\ http://www.merl.com
}

\title{
A Hybrid UWB Modulation Design Compatible for Both Coherent and Transmit-Reference Receivers
}

Philip Orlik, Andreas Molisch

TR2005-087 April 2006

\begin{abstract}
In a pulsed UWB system, either coherent receivers or transmit-reference (TR) receivers can be used to demodulate the signals. For coherent receivers, which are typically based on a Rake Structure, the large number of resolvable multipaths is a major challenge for channel estimation and receiver complexity. TR receivers can effectively collect energy from all the received multipath components with much smaller complexity, but show reduced performance. Current modulation formats are tuned to either coherent or TR receivers, but cannot interoperate with both of them. In this paper, we propose an innovative modulation scheme that enables the useage of TR and coherent recievers in the same wireless network. The modulation allows demodulation by TR receivers, while at the same time enabling a coherent receiver to fully exploit all available signal energy, and actually to perform slightly better (by $1.8 \mathrm{~dB}$ ) than a coherent receiver working with conventional BPSK modulation; this additional gain can be interpreted as a coding gain of a trellis-coded modulation scheme. The scheme thus allows the network designers to trade off performance vs. complexity in the receivers, while allowing a uniform signaling (modulation) scheme for all transmitters.
\end{abstract}

ICC 2006

This work may not be copied or reproduced in whole or in part for any commercial purpose. Permission to copy in whole or in part without payment of fee is granted for nonprofit educational and research purposes provided that all such whole or partial copies include the following: a notice that such copying is by permission of Mitsubishi Electric Research Laboratories, Inc.; an acknowledgment of the authors and individual contributions to the work; and all applicable portions of the copyright notice. Copying, reproduction, or republishing for any other purpose shall require a license with payment of fee to Mitsubishi Electric Research Laboratories, Inc. All rights reserved.

Copyright (C) Mitsubishi Electric Research Laboratories, Inc., 2006

201 Broadway, Cambridge, Massachusetts 02139 



\title{
A Hybrid UWB Modulation Design Compatible for both Coherent and Transmit-Reference Receivers
}

\author{
Philip Orlik ${ }^{1}$ Member IEEE, Shiwei Zhao ${ }^{1,2}$, Student Member IEEE, Andreas F. Molisch ${ }^{1, \dagger}$ Fellow IEEE \\ ${ }^{1}$ Mitsubishi Electric Research Labs, 201 Broadway Ave., Cambridge, MA, 02139 \\ ${ }^{2}$ School of Electrical Engineering and Computer Science Oregon State University, Corvallis, OR 97331 \\ E-mail:porlik,molisch@merl.com, zhao@engr.orst.edu
}

\begin{abstract}
In a pulsed UWB system, either coherent receivers or transmit-reference (TR) receivers can be used to demodulate the signals. For coherent receivers, which are typically based on a Rake structure, the large number of resolvable multipaths is a major challenge for channel estimation and receiver complexity. TR receivers can effectively collect energy from all the received multipath components with much smaller complexity, but show reduced performance. Current modulation formats are tuned to either coherent or TR receivers, but cannot interoperate with both of them at the maximum performance possible for each of them. In this paper, we propose an innovative modulation scheme that enables the use of $T R$ and coherent receivers in the same wireless network. The modulation allows demodulation by TR receivers, while at the same time enabling a coherent receiver to fully exploit all available signal energy, and actually to perform slightly better (by $1.8 \mathrm{~dB}$ ) than a coherent receiver working with conventional BPSK modulation; this additional gain can be interpreted as a coding gain of a trellis-coded modulation scheme. The scheme thus allows the network designers to trade off performance vs. complexity in the receivers, while allowing a uniform signaling (modulation) scheme for all transmitters.
\end{abstract}

Index Terms - Ultra-wideband, transmit-reference, coherent, RAKE, trellis.

\section{INTRODUCTION}

While ultrawideband signals have been applied in radar for a long time, their use for wireless communications is relatively recent. In February 2002, the American FCC (Federal Communications Commission) allowed the use of UWB systems for communications in the $3.1-10.6 \mathrm{GHz}$ band if certain restrictions with respect to bandwidth and power spectral density are fulfilled [1].

The most widely-studied scheme for UWB transmission is time-hopping impulse radio (TH-IR), first suggested in the pioneering work of Win and Scholtz [2]. In this scheme, each symbol is represented by a number of pulses, whose position is determined by a pseudorandom time-hopping sequence, which can differ from user to user. The original TH-IR scheme used pulse position modulation; however, in recent years, binary phase shift keying (BPSK), combined with polarity scrambling, has become more popular [3] [4].

One advantage of the large signal bandwidth in UWB systems is the robustness to multipath fading that can be achieved due to the fine multipath resolution that is seen by the large bandwidth signal. A common approach to energy capture

$\dagger$ A. F. Molisch is also at the Department of Electroscience, Lund University, Sweden in the presence of multipath propagation is to implement a RAKE receiver [5], [6], [7]. However, in a pulse-based UWB system, the number of resolvable paths could reach tens to over a hundred in typical indoor propagation environments [8], [9], [10], which imposes technical hurdles as well as implementation difficulties. In order to capture a considerable portion of the signal energy scattered in the multipath components, a receiver based on a digital Rake structure has to sample (and process the samples) at a minimum of hundreds of $\mathrm{MHz}$ to even multi-GHz clock rates, or requires an impractically large number of Rake fingers. Thus realizing optimal RAKE reception performance requires accurate channel and timing knowledge, which is quite challenging to obtain as the number of resolvable paths grows. Moreover, the received pulse shapes of resolvable multipath are distorted differently due to diffraction, which make it suboptimal to use the transmitted pulse waveform as the correlation template in RAKE reception, [11]. Because of these issues, which are unique to UWB spread spectrum, the popular optimal RAKE receiver design can become rather difficult. Reduced-complexity Rake receivers have been proposed [7] but naturally lead to a loss in performance.

As an alternative, Transmit-Reference impulse radio (TRIR) systems eliminate the need for a RAKE receiver [12], [13]. In a TR-IR system, the information is encoded as phase differences between successive pulses (notice the difference to differential modulation, where the information is encoded in the phase difference between successive symbols). Thus, each symbol in a TR-IR system is a sequence of time-hopped 'doublets' or pairs of two consecutive pulses. Typically, the first pulse in the pair is referred to as a reference pulse, and is not modulated; the second pulse, which is modulated by the data, is referred to as a data pulse. The two pulses in each pair are separated by a fixed delay $T_{d}$. In a time-hopping system with TR, it is now those doublets that are transmitted at pseudorandom delays. At the receiver, the received signal just needs to be multiplied with a delayed (by duration $T_{d}$ ) version of itself, and integrated, thus obviating the need for a Rake receiver. However, the reduced complexity is bought at the cost of requiring twice the number of pulses, and the additional energy required for the reference pulses, typically $3 \mathrm{~dB}$.

The main drawback of a TR scheme stems from a reduced signal-to-noise ratio (SNR). This reduction is partly due to "wasting" energy on the reference pulses that are not carrying any information. More importantly, the differential detection 
gives rise to excess noise related to the multiplication of noise contributions in the received reference pulses with the noise contributions in the received data pulses. Those cross-terms are especially troublesome in a UWB receiver: the input to the multiplication operator has a very low SNR, because the signal has not undergone a despreading operation. This results in large noise-noise terms that are integrated over a certain time interval. The authors in [13], [14] suggested averaging the reference pulses over several pulse durations, in order to reduce the noise in the reference pulses, and thus also the cross-terms. The important paper of [13] also gives a detailed derivation of the error probability for both the conventional TR receiver and the receiver that uses averaged reference pulses.

Theoretically, it is possible to demodulate a TR signal with a coherent receiver, by simply "throwing away" the reference pulses. However, that implies a $3 \mathrm{~dB}$ penalty compared to a system whose modlation is designed for the use with coherent receivers, e.g. BPSK. On the other hand, a signal that is designed for coherent receivers (i.e., without the reference pulses) can obviously not be demodulated with a TH-IR receiver. Thus, the decision to use either coherent or TR-IR modulation leads to incompatible system structures. However, for many applications in sensor networks and similar communications applications, it is desirable to enable heterogeneous networks where different nodes with different cost, complexity and performance trade-offs exist within the same network. This is especially critical if multicast or broadcast is to be used within such a network.

Therefore, we propose in this paper a new modulation technique that enables both coherent RAKE and TR-IR transceivers to co-exist in the same wireless network, without performance penalty for the coherent receivers. Our idea is based on our observation that TR-IR systems encode an information bit as a phase difference between a reference pulse and a data pulse, but that the polarity of the reference pulse is inconsequential for the correct operation of the TR-IR system. Therefore, we encode redundant information on the reference pulses as well. Therefore, a coherent receiver can decode the information with improved performance, while the modulation maintains the required phase difference between the data pulse and reference pulse, so that a TR-IR receiver can also decode the information. We call this modulation 'hybrid-IR' (H-IR).

The remainder of the paper is organized the following way: Section II presents a system overview that points out the most salient features of our new modulation format. Section III then explains details about several enhancements of the basic ideas, and describes receiver structures that can be used in conjunction with the mandatory aspects. Section IV finally presents the coding gain and the performance simulations for our proposed system. The paper is summarized in Section V.

\section{SySTEM MODEL}

In the proposed modulation format, each bit or symbol is represented by $N_{f}$ pulse doublets, where $N_{f}$ is a positive integer. Time-hopping (TH) and polarity scrambling are applied on each doublet within the symbol to obtain processing gain to combat multiple-access interference (MAI) and flatten

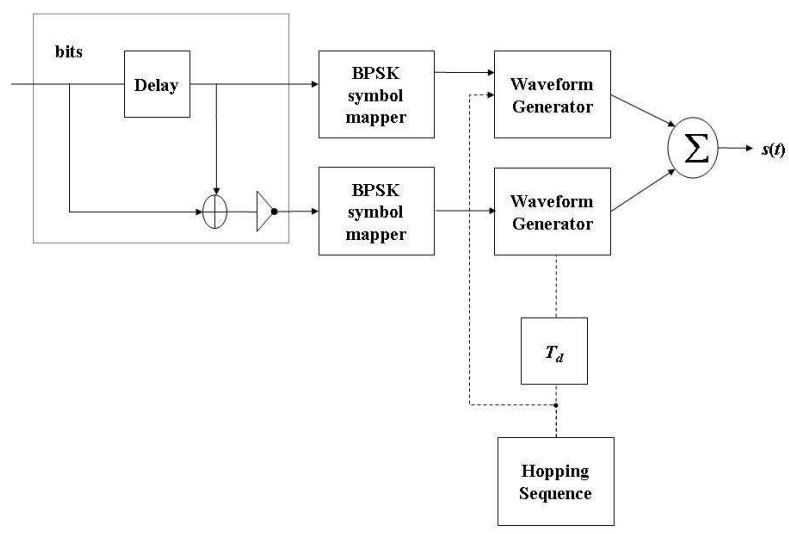

Fig. 1. Block diagram of a hybrid-IR transmitter.

the signal spectrum. The time taken to transmit a bit is $T_{s}$, which is called the symbol duration. The time $T_{s}$ is partitioned into "frames" $T_{f}$, and the frames are partitioned into chips $T_{c}$ corresponding typically to a pulse duration. If $N_{c}$ represents the number of chips in a frame and $N_{f}$ represents the number of frames in a symbol, then $T_{s}, T_{f}$, and $T_{c}$ are related as follows

$$
T_{s}=N_{f} T_{f}=N_{f} N_{c} T_{c} .
$$

As discussed in Sec. I, the key idea of our modulation scheme is to encode information on the reference pulse. Specifically, we suggest that the polarity of the reference pulse of the $i$-th symbol is determined by the value of the $i-1$ th bit. Fig. 1 shows a H-IR transmitter according to this principle. The transmitter includes a pre-processor for input bits. The pre-processor includes a delay and an adder. The adder sums each input bit with the previous bit of the input stream. The sum is then inverted. The pre-processing generates a pair of modulation symbols from two successive information bits. During each symbol period, the symbols are modulated using BPSK modulators. The reference pulses in the sequence are BPSK modulated according to the input bits, and data pulses are BPSK modulated according to the inverted sum. Both sequences undergo the time-hopping, the data pulses are delayed by $T_{d}$, and the sequences are added up. The transmitted waveform, $s(t)$, thus has the mathematical form

$$
\begin{aligned}
s(t)= & \sum_{i=0}^{\infty} \sum_{j=0}^{N_{f}-1} d_{i, j}\left\{b_{i-1} p\left(t-i T_{s}-j T_{f}-c_{j} T_{c}\right)\right. \\
& \left.+\left(\overline{b_{i-1} \oplus b_{i}}\right) p\left(t-i T_{s}-j T_{f}-c_{j} T_{c}-T_{d}\right)\right\}
\end{aligned}
$$

where $b_{i}$ is the $i^{t h}$ modulation symbol and $c_{j}$ is the $j^{\text {th }}$ value of the TH code, in the range $\left\{0,1, \ldots, N_{T H}\right\} . N_{T H}$ is smaller than $N_{c}$ and $N_{c} T_{c}=N_{T H} T_{c}+T_{d}+T_{g}$, where $T_{g}$ is a guard time set to protect from inter-frame interference. Additionally an optional polarity scrambling sequence $d_{i, j} \in \pm 1$ can be applied to each doublet in the transmitted signal so as to flatten the signal spectrum.

The modulation according to Eq. (2) shows that the phase difference between the reference pulse and data pulse is 
identical to that of a conventional TR-IR system. Table I shows the four possible combinations of a previous and a current bit, the corresponding values of the reference and data waveforms, and their phase differences or polarities.

TABLE I

REFERENCE AND DATA PULSE MODULATION SYMBOL MAPPING

\begin{tabular}{|l||l||l||l||l|}
\hline $\begin{array}{l}\text { Previous } \\
\text { bit }\end{array}$ & $\begin{array}{l}\text { Current } \\
\text { bit }\end{array}$ & $\begin{array}{l}\text { Reference } \\
\text { modu- } \\
\text { lation } \\
\text { symbol } \\
b_{i-1}\end{array}$ & $\begin{array}{l}\text { Data modulation } \\
\text { symbol } \frac{b_{i-1} \oplus b_{i}}{}\end{array}$ & $\begin{array}{l}\text { Reference, } \\
\text { data pulse } \\
\text { phase } \\
\text { difference }\end{array}$ \\
\hline 0 & 0 & -1 & 1 & $180^{\circ}$ \\
\hline 0 & 1 & -1 & -1 & $0^{\circ}$ \\
\hline 1 & 0 & 1 & -1 & $180^{\circ}$ \\
\hline 1 & 1 & 1 & 1 & $0^{\circ}$ \\
\hline
\end{tabular}

If the current bit is ' 0 ', then the phase difference between the reference pulse and the data pulse is always $180^{\circ}$, regardless of the value of the previous bit. If the current bit is ' 1 ', then the phase difference is $0^{\circ}$. Additionally, the sequence of pairs also contains the information about the previous bit in the polarity of the reference pulse. Again, this is seen in Table I, where the reference pulse in each pair has a positive polarity if the previous bit was a ' 1 ', and a negative polarity when the previous bit was a ' 0 '.

This waveform, therefore, enables the use of both coherent and differentially coherent receivers in the same network. A TR-IR receiver operates by simply delaying the received signal, $r(t)$, by the doublet delay, $T_{d}$, and correlating this delayed signal with the original $r(t)$ over the symbol frames. The TR-IR receiver then computes a decision statistic for the $i^{\text {th }}$ bit, $z_{i}^{T R}$ according to

$$
z_{i}^{T R}=\sum_{j=0}^{N_{f}-1} \int_{i T_{s}-j T_{f}-c_{j} T_{c}}^{i T_{s}-j T_{f}-c_{j} T_{c}+T_{I}} r(t) r\left(t-T_{d}\right) d t
$$

In the above $T_{I}$ is the integration interval which is typically set based on the delay spread of the channel. The TR-IR receiver then decides whether a ' 0 ' or ' 1 ' was sent based on the sign of $z_{i}^{T R}$

As outlined above, a TR-IR receiver can demodulate the signal. However, the signal can also be demodulated by a coherent $\mathrm{TH}$ receiver with improved performance (compared to the coherent reception of TR-IR signals). The gain in performance is based on the fact that information is encoded in both the reference pulses and the data pulses. Thus, the coherent $\mathrm{TH}$ receiver can use the energy in the reference pulses to make decisions on the values of the transmitted bits, see Table I.

\section{Alternative INTERPRETATION AND RECEIVER STRUCTURE}

In the following, we give another interpretation of the modulation format, which also leads to a suggested receiver structure.
During each symbol period, a sequence of $N_{f}$ pulse pairs is transmitted. The pair in each frame consists of two pulses, each with a polarity depending on the current and previous bit transmitted. There are four possible combinations of pairs.

$$
\begin{aligned}
& s_{0}(t)=-1 * \frac{1}{\sqrt{2 N_{f} E_{p}}} p(t)+1 * \frac{1}{\sqrt{2 N_{f} E_{p}}} p\left(t-T_{d}\right) \\
& s_{1}(t)=-1 * \frac{1}{\sqrt{2 N_{f} E_{p}}} p(t)-1 * \frac{1}{\sqrt{2 N_{f} E_{p}}} p\left(t-T_{d}\right) \\
& s_{2}(t)=1 * \frac{1}{\sqrt{2 N_{f} E_{p}}} p(t)-1 * \frac{1}{\sqrt{2 N_{f} E_{p}}} p\left(t-T_{d}\right)(4) \\
& s_{3}(t)=1 * \frac{1}{\sqrt{2 N_{f} E_{p}}} p(t)+1 * \frac{1}{\sqrt{2 N_{f} E_{p}}} p\left(t-T_{d}\right)
\end{aligned}
$$

The coefficient $\frac{1}{\sqrt{2 N_{f} E_{p}}}$ in the above equations normalizes the transmitted symbol to unit energy, where $E_{p}$ is the energy of the pulse, and $N_{f}$ is the number of pulse pairs in a symbol. We can also represent the signals as a vector

$$
\begin{aligned}
& s_{0}=\left[\begin{array}{rr}
-1 & 1
\end{array}\right] \\
& s_{1}=\left[\begin{array}{rr}
-1 & -1
\end{array}\right] \\
& s_{2}=\left[\begin{array}{rr}
1 & -1
\end{array}\right] \\
& s_{3}=\left[\begin{array}{rr}
1 & 1
\end{array}\right]
\end{aligned}
$$

Therefore, the transmitted signal can also be described as follows. During each symbol period, the transmitter transmits a sequence of $N_{f}$ pairs. The four possible pairs are given by Eq. (4). The pairs could optionally be scrambled with a polarity code in order to flatten the spectrum.

Under this interpretation of the hybrid modulation format, we see that it provides a modulation format with memory. Modulation formats that have memory can be represented by a trellis diagram. Additionally, the transmitted signals are now two-dimensional signals because two orthogonal basis functions $\Psi_{0}(t)=\frac{1}{\sqrt{2 N_{f} E_{p}}} p(t)$ and $\Psi_{1}(t)=\frac{1}{\sqrt{2 N_{f} E_{p}}} p\left(t-T_{d}\right)$ are used to represent the pulse pairs.

Figure 2 shows a trellis diagram describing the modulation. The trellis has two states, where a state 0 occurs if the value of the previous bit is ' 0 ', and state 1 if the value of a previous bit is '1'. Branches of the trellis indicate possible transitions. The branches are labeled with the value of current input bit, and the vector representation of the transmitted pair. For example, if the current state is 0 and a ' 1 ' bit is to be transmitted, then a transition to state 1 occurs, and the pulse pair $s_{1}=[-1-1]$ is transmitted.

With this interpretation of the H-IR modulation, we see that a coherent RAKE receiver can be used to demodulate the signal. Fig. 3 shows the RAKE receiver structure according to the proposal. Now the RAKE fingers correlate the incoming signals with sequences of the two basis pulses, $\Psi_{0}(t)$ and $\Psi_{1}(t)$. The output of each finger is now a 2-D vector. The outputs of the fingers are combined to produce a soft input observations for a conventional maximum likelihood sequence detector (MLSD). The MLSD detector determines a most probable path through the trellis for a given sequence of observations. Methods that approximate the MLSD detector, such as Viterbi decoding [15] can also be used. 


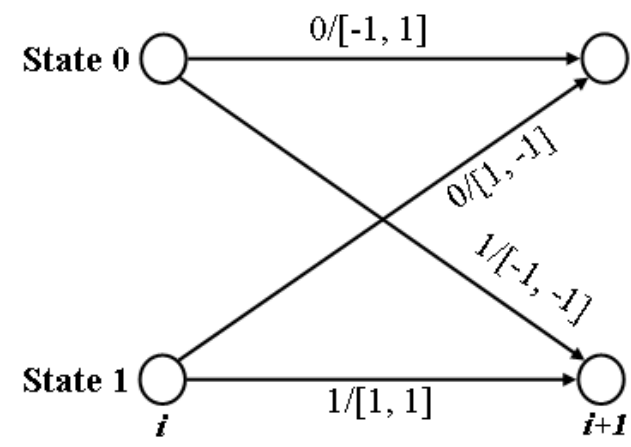

Fig. 2. Trellis representation of the hybrid modulation.

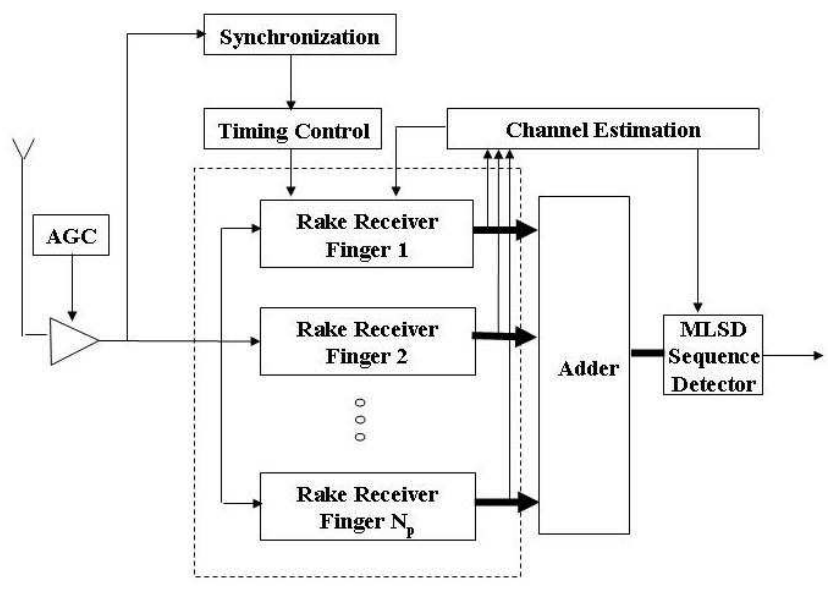

Fig. 3. Coherent RAKE receiver for hybrid modulation.

\section{Receiver Performance ANd Numerical Results}

A coherent receiver that demodulates a TR signal by simply "throwing away" the reference pulses suffers from a $3 \mathrm{~dB}$ performance penalty compared with an uncoded, coherently detected BPSK signal. We show in the following that our modulation scheme not only allows the recovery of those 3 $\mathrm{dB}$, but also gives an additional coding gain.

If we assume $\varepsilon$ is the bit energy, a BPSK signal constellation $\{ \pm \sqrt{\varepsilon}\}$ and a conventional TR signal constellation $\{ \pm \sqrt{\varepsilon / 2}\}$ (ignoring the reference pulse) have the Euclidean distances of $d_{0}=2 \sqrt{\varepsilon}$ and $d_{1}=\sqrt{2 \varepsilon}$ separately. Consider now the free Euclidean distance of the trellis in Fig. 2. The hybrid trellis modulation can be viewed as a systematic convolutional encoder or a trellis coded modulation (TCM). From Fig. 2 the minimum Euclidean distance between any two signal paths that diverge from one state and merge again at the same state is $\sqrt{d_{0}^{2}+d_{1}^{2}}=\sqrt{6 \varepsilon}$. This means that this trellis modulation not only compensates the $3-\mathrm{dB}$ loss incurred by transmitting reference pulses in TR signaling, but also gives an additional coding gain of about $1.76 \mathrm{~dB}$ compared with uncoded BPSK signaling. In addition, the trellis decoding does not impose a significant increase in complexity since it is only a very simple two-state trellis. In the following we validate the claimed performance improvement by simulations.

The results presented in this section used a carriermodulated, truncated root-raised-cosine (RRC) pulse with a roll-off factor 0.25 as the UWB pulse shape $p(t)$ and the
$10 \mathrm{~dB}$ signal bandwidth is $500 \mathrm{MHz}$. We use the standardized IEEE 802.15.4a channel model [19], which is the most detailed and comprehensive UWB channel models currently available. The channel model is generated from experimental measurements in different typical communication environments, including residential, office, industry, outdoor, etc, covering the frequency range from 2 to $10 \mathrm{GHz}$. The channel model is specified in a complex baseband representation, as is the simulation we performed. For multipath results perfect channel state information was assumed to be available at the receiver. The chip duration, $T_{c}$, was set at $4 \mathrm{nsec}$, and the pulse pair spacing $T_{d}$ and the guard time $T_{g}$ were both $20 \mathrm{nsec}$. The data rate is set as $1 \mathrm{Mbps}$. The TH sequence was constructed based on the algorithm proposed in [18] with $N_{T H}=N_{f}=11$.

Since the TR receiver has been analyzed intensively in literature [12]-[14], [16], [17], here we focus on how this innovative signaling format performs with the coherent RAKE MRC receiver. For a reasonable low hardware complexity, 10 rake fingers are employed to collect the 10 strongest paths of each received symbol. Outputs from the MRC are fed into the MLSD detector and demodulated based on the trellis shown in Fig. 2.

Fig. 4 presents the system performance for AWGN channels and compares it with the theoretical performance of BPSK modulated system. The system performance under multipath fading channel is given in Fig. 5. The measured channel impulse response model in non-line-of-sight (NLOS) industrial environments (Channel model 8) from [19] is used in the simulation. For comparison, a BPSK modulated system is simulated with the same channel and uses the same processing gain, same time hopping and polarity scrambling sequences. The gain of H-IR over BPSK modulation is clearly illustrated.

To complete the performance simulations and to demonstrate the use of a TR receiver with H-IR modulation, we investigated the BER of a TR (delay and correlate) when it is applied to H-IR modulation. The BER under an AWGN channel is shown in Fig. 6. Here we see the loss relative to uncoded coherent BPSK, due to both the energy used in transmitting the reference pulse and the noise-noise cross terms that degrade the receivers SNR. While the performance is significantly worse than that of a coherent receiver, the complexity of the TR receiver is also significantly reduced since no RAKE is employed.

\section{CONCLUSION}

In this paper we introduced a modulation format that enables flexible receiver design, in that the signal can be demodulated using either a coherent receiver, e.g. a RAKE, or a differentially coherent receiver, e.g., delay and correlate. The advantage of the proposed scheme is that coherent receivers are not penalized the $3 \mathrm{~dB}$ that they would have lost if they were employed to receive a conventional TR signal. In fact a modest coding gain is achieved by the coherent receiver due to the exploitation of the memory inherent in the proposed H-IR modulation format. This improves the coherent receiver performance by a coding gain of $4.76 \mathrm{~dB}$ over conventional coherent reception of TR signaling and $1.76 \mathrm{~dB}$ over uncoded BPSK signaling. 


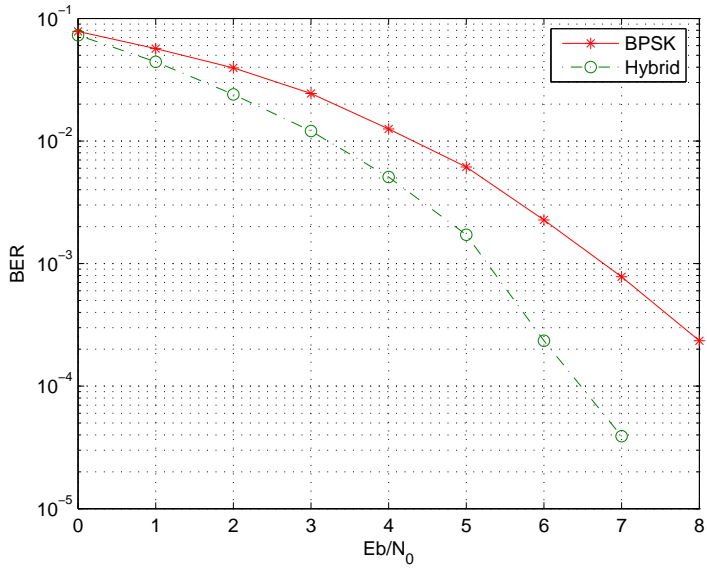

Fig. 4. BER versus SNR curves of coherent H-IR receiver versus theoretical performance in an AWGN channel.

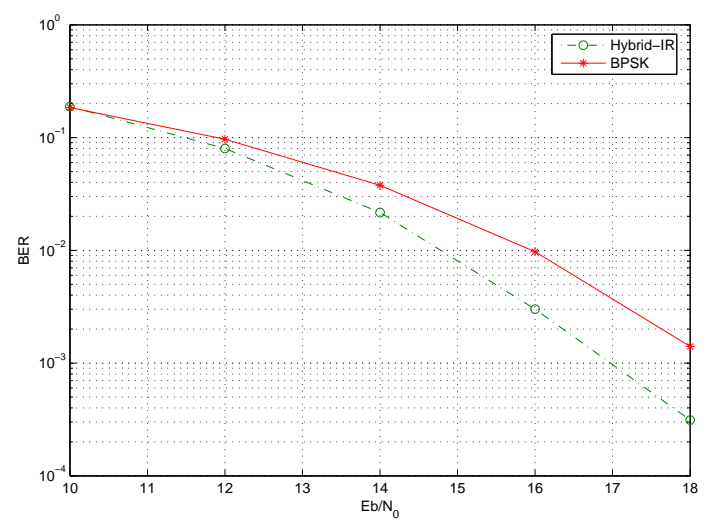

Fig. 5. BER versus SNR curves of coherent rake receiver using H-IR signaling in a multipath channel (802.15.4a CM 8 industrial non-line-of-sight).

\section{REFERENCES}

[1] FCC notice of proposed rule making, "Revision of part 15 of the commission's rules regarding ultra-wideband transmission systems," ETDocket 98-153.

[2] M. Win, and R.A. Scholtz, "Ultra-wide bandwidth time-hopping spreadspectrum impulse radio for wireless multiple -access communications," IEEE Trans. Comm., vol. 48, pp. 679-691, April 2000.

[3] Y. P. Nakache and A. F. Molisch, "Spectral Shape of UWB Signal Influence of Modulation Format, Multiple Access Scheme and Pulse Shap", Proc. 57th IEEE Vehicular Techn. Conf., spring, pp. 2510-2514 2003.

[4] E. Fishler and H. V. Poor, "On the Tradeoff Between Two Types of Processing Gains", IEEE Trans. Comm., vol 53, pp. 1744-1753, Sept. 2005.

[5] M.Z. Win and R.A. Scholtz, "On the energy capture of ultrawide bandwidth signals in dense multipath environments," IEEE Communications Letters, vol.2, no.9, pp.245-247, Sept. 1998.

[6] S. Gaur, A. Annamalai, "Improving the range of UWB transmission using RAKE receivers," Proc. of the 53rd IEEE VTC, vol. 1, pp. 597-601, Oct. 2003.

[7] D. Cassioli and M. Z. Win and A. F. Molisch and F. Vatelaro, "Performance of selective Rake reception in a realistic (UWB) channel", Proc. ICC 2002, pp. 763-767, 2002.

[8] A. F. Molisch, J. R. Foerster, and M. Pendergrass, "Channel models for ultrawideband personal area networks," IEEE wireless communications, vol. 10, No. 6 Dec. 2003.

[9] J. Karedal and S. Wyne and P. Almers and F. Tufvesson and A. F. Molisch,

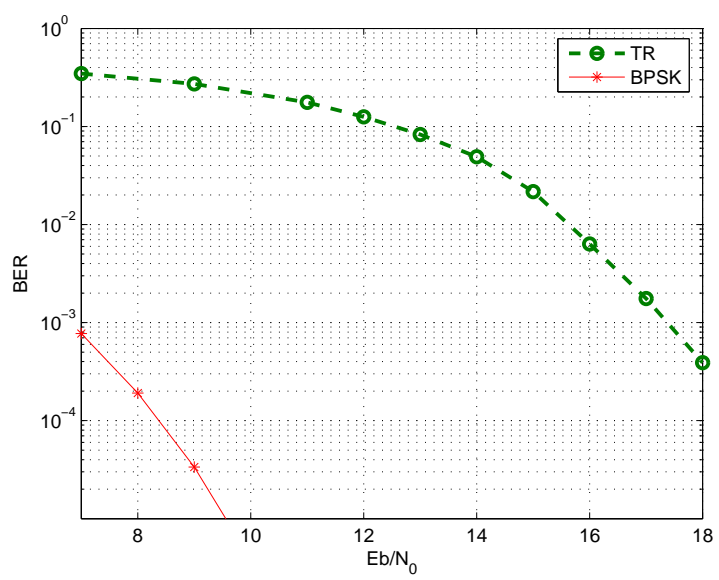

Fig. 6. BER versus SNR, H-IR signalling and differentially coherent receiver.

"Statistical Analysis of the UWB Channel in an Industrial Environment," Proc. IEEE VTC fall 2004, pp. 81-85, 2004.

[10] A. F. Molisch, "Ultrawideband propagation channels - theory, measurement, and models," IEEE Trans. Vehicular Techn., special issue on UWB, invited paper, pp. 1528-1545 2005.

[11] R. C. Qiu, "A study of the ultra-wideband wireless propagation channel and optimum uwb receiver design", IEEE J. Selected Areas Comm., vol. 20 , pp. $1628-1637$ (2002).

[12] R. T. Hoctor and H. W. Tomlinson, "An overview of delay-hopped, transmitted-reference RF communications," in Technical Information Series, G.E. Research and Development Center, pp. 1-29, Jan. 2002.

[13] J. D. Choi and W. E. Stark, "Performance of ultra-wideband communications with suboptimal receiver in multipath channels," IEEE Journal on Selected Areas in Communications, vol. 20, No. 9, pp. 1754-1766, Dec. 2002.

[14] L. Yang and G. B. Giannakis, "Optimal pilot waveform assisted modulation for ultra-wideband communications," IEEE Transactions on Wireless Communications, vol.3, no. 4, pp. 1236-1249, July 2004.

[15] A. F. Molisch, Wireless Communications. IEEE-Press Wiley, 2005.

[16] S. Gezici, F. Tufvesson, and A.F. Molisch, "On the performance of transmitted-reference impulse radio," Proc. of IEEE Globecom'04, vol. 5, pp. 2874-2879, Nov. 2004.

[17] S. Zhao, H. Liu, and Z. Tian, "A decision-feedback authcorrelation receiver for pulsed ultra-wideband systems," Proc. of IEEE 2004 Radio and Wireless Conference (Rawcon'04), pp. 251-254, Sept. 2004.

[18] R.A. Scholtz, P.V. Kumar, and C. J. Corrada-Bravo, "Signal Design for Ultra-wideband Radio," Sequences and Their Applications (SETA 01), 2001.

[19] A.F. Molisch, K. Balakrishnan, C.-C. Chong, S. Emami, A. Fort, J. Karedal, J. Kunisch, H. Schantz, U. Schuster, K. Siwiak, "IEEE 802.15.4a channel model - final report." 